\title{
A REMARK ON LATIN SQUARES AND BLOCK DESIGNS
}

\author{
W. D. WALLIS \\ (Received 12 November 1969; revised 19 December 1969) \\ Communicated by G. Szekeres
}

1

Throughout this paper any matrix is square and of order $q$ unless otherwise specified. $I$ and $J$ will represent an identity matrix and a square matrix with every element +1 respectively; if necessary, the size will be indicated by a subscript. The Kronecker product of matrices $A=\left(a_{i j}\right)$ and $B$ is the block matrix whose $(i, j)$-th block is $a_{i j} B$, and is written $A \times B$.

Balanced incomplete block designs, $(v, k, \lambda)$-configurations and mutually orthogonal Latin squares are defined, for example, in [2]. The following result is Lemma 6 of [3]:

Lemma 1. Suppose $k$ and $q$ are positive integers with $k<q$, such that a $(q, k, \lambda)$-configuration exists and such that there is $a(0,1)$ matrix $Q$ of dimension $k q \times q^{2}$ satisfying

$$
\left\{\begin{array}{l}
Q Q^{T}=q I_{k q}+\left(J_{k}-I_{k}\right) \times J \\
J_{k q} Q=k J^{*} \\
Q J_{q^{2}}=q J^{*}
\end{array}\right.
$$

where $J^{*}$ is the matrix of size $k q \times q^{2}$ with every element +1 . Then there is a balanced incomplete block design with parameters

$$
b=q\left(k^{2}+\lambda\right), \quad v=q k, \quad r=k^{2}+\lambda, k, \lambda .
$$

We shall show that the existence of (at least) $k-2$ mutually orthogonal Latin squares of order $q$ is equivalent to the existence of $Q$; this proves

THEOREM 2. If there exist $k-2$ mutually orthogonal squares of order $q$ and a $(q, k, \lambda)$-configuration, then there is a block design with parameters (2).

Theorem 2 of [3] is essentially our Theorem in the particular case when $q$ is a prime power.

The author is indebted to his referee, who pointed out the equivalences in Section 2 and suggested other improvements which have shortened and strengthened the paper. 
Suppose $Q$ is a $(0,1)$ matrix of order $k q \times q^{2}$. We will show that the first line of condition (1) implies the other two parts. Partition $Q$ into $k$ blocks, $B_{1}, \cdots, B_{k}$ of size $q \times q^{2}$. The first part of (1) then becomes

$$
\begin{array}{ll}
B_{i} B_{i}^{T}=q I & i=1,2, \cdots, k \\
B_{i} B_{j}^{T}=J & 1 \leqq i<j \leqq k .
\end{array}
$$

We show that (a) and (b) are together equivalent to conditions (3), (4) and (5):

(3) each row of $Q$ contains exactly $q$ elements 1 ;

(4) each column of any $B_{i}(i=1,2, \cdots, k)$ contains exactly one element 1 ;

(5) given any two rows from different blocks, there is one and only one column of $Q$ containing an entry 1 in each of the rows.

It is clear that (b) and (5) are equivalent; obviously (a) implies (3) - consider the diagonal elements of $B_{i} B_{i}^{T}$. If (a) is true then each $B_{i}$ contains exactly $q^{2}$ elements 1, using (3); if two of them are in the same column, in rows $x$ and $y$ of $B_{i}$ say, then the $(x, y)$ element of $B_{i} B_{i}^{T}$ will be non-zero, contradicting (a); so (4) follows. Conversely, (3) and (4) together immediately imply (a).

Now (3) is just the last line of (1), and (4) implies the second part of (1). So condition (1) is equivalent to its first line, which is in turn equivalent to (3), (4) and (5).

The existence of a set of $k-2$ mutually orthogonal Latin squares of order $q$ is equivalent [2, p. 82] to the existence of a $k \times q^{2}$ array $A=\left(a_{i j}\right)$, whose elements $a_{i j}$ are chosen from the symbols $1,2, \cdots, \boldsymbol{q}$ and are arranged in such a way that the columns of any $2 \times q^{2}$ subarray are the $q^{2}$ ordered pairs obtainable from $\{1,2, \cdots, \boldsymbol{q}\}$.

Suppose $A$ exists. Corresponding to the $i$-th row of $A$ form a $(0,1)$ matrix $B_{i}$ of size $q \times q^{2}$ such that the $(r, t)$ element of $B_{i}$ is 1 if and only if $a_{i t}=\boldsymbol{r}$. Then choose $Q$ to be the column $B_{1}, B_{2}, \cdots, B_{k}$. Since $r$ occurs $q$ times in each column of $A$, this $Q$ satisfies (3); as $a_{i t}$ has a unique value, we have (4). Row $r$ of $B_{i}$ and row $s$ of $B_{j}$ will both have 1 in column $t$ if and only if $\left(a_{i t}, a_{j t}\right)=(r, s)$, and by the definition of $A$ there is exactly one such $t$ for given $i$ and $j$; so $Q$ satisfies (5).

Conversely, suppose there is a matrix $Q$ satisfying (3), (4) and (5). Define $a_{i t}=r$ where the $(r, t)$ element of $B_{i}$ is 1 . By (4) $r$ is uniquely defined; (3) and (5) show that $A$ satisfies the conditions at the beginning of this section.

We have proven the remark which preceded Theorem 2; the Theorem follows.

Suppose there exists a balanced incomplete block design with $\lambda=1$ and $k$ the parameter of a projective plane. In this case E. T. Parker proved [1, Theorem 
1] that there exists a set of $k-2$ mutually orthogonal Latin squares of order $v$. A little arithmetic shows that we can use this together with our Theorem 2 only when the block design in Parker's Theorem is symmetric and has the same parameters as the configuration required in Theorem 2; we then have the following result.

COROLLARY. If there exist projective planes of parameters $k$ and $k-1$, then there is a balanced incomplete block design with parameters.

$$
b=\left(k^{2}-k+1\right)\left(k^{2}+1\right), \quad v=k\left(k^{2}-k+1\right), \quad r=k^{2}+1, k, 1 .
$$

For example, designs (6) exist for $k=5$ and 8.

\section{References}

[1] E. T. Parker, 'Construction of some sets of mutually orthogonal Lalin squares', Proc. Amer. Math. Soc. 10, (1959), 946-949.

[2] H. J. Ryser, Combinatorial Mathematics (Carus Monograph 14, M.A.A. 1963).

[3] Jennifer Wallis, 'Some results on configurations', Journal Australian Math. Soc. 12 (1971), $378-384$.

Mathematics Department

University of Newcastle

New South Wales 